\title{
A sounding rocket payload experiment on zero gravity fuel gauging using modal analysis
}

\author{
Kevin M. Crosby ${ }^{1, \dagger}$, Eric Ireland ${ }^{1}$, Kevin Lubick ${ }^{1}$, Steven Mathe ${ }^{1}$, Steven Metallo ${ }^{1}$, Rudy Werlink ${ }^{2}$ \\ ${ }^{1}$ Carthage College, Kenosha, WI, USA ${ }^{2}$ NASA Kennedy Space Center, Florida, USA \\ (rudy.werlink-1@nasa.gov) ${ }^{\dagger}$ \\ Corresponding author: kcrosby@ carthage.edu
}

\begin{abstract}
For the past three years Carthage Space Sciences faculty and students have been developing Experimental Modal Analysis (EMA) techniques for use in microgravity fuel gauging of spacecraft propellant tanks. Our experiments were initially conducted on parabolic flights in which periods of microgravity of 20 seconds can be obtained. Parabolic flights were necessary to demonstrate the effectiveness of the technique in a relevant environment, but the short period of microgravity available during each parabola is insufficient to probe the resolution of the technique for settled, non-sloshing propellant. Here we report the results of an experiment in which the EMA technique is implemented in a sounding rocket payload developed through participation in the RockSat-C program of the Colorado and Virginia Space Grant Consortia.
\end{abstract}

\section{Introduction}

Low gravity propellant mass gauging is identified in NASA's Exploratory Systems Architecture Study as a critical path objective [NASA, 2005]. The future of manned spaceflight beyond LEO relies in part on the development of accurate and robust methods of mass gauging in both settled and unsettled propellant states.

Traditional methods of fuel gauging require the presence of an external gravity field. Such methods typically rely on a buoyant force acting on a surface level indicator (e.g., the fuel gauge used in automobile gas tanks) or a measurement of effective capacitance of a probe in partial contact with the liquid. Such methods are not effective under microgravity conditions where the liquid surface position and shape is unrelated to the volume of liquid in the tank.

Fuel volume estimation on spacecraft instead use indirect gauging methods including equationof-state estimations (for pressurized systems), measurement of spacecraft dynamics, and burntime integration. Equation of state estimations require that the propellant tank be pressurized by a known volume of pressurant (typically helium). The pressure and temperature of the ullage volume in the tank is monitored as propellant is burned and the volume of the ullage and therefore of the remaining propellant is calculated based on the appropriate equation of state (usually the ideal gas law). This method, while effective, requires the additional hardware of pressurant tanks, pumps, valves and other hardware necessary to transfer pressurant to the propellant tanks. At launch costs exceeding $\$ 10,000 / \mathrm{kg}$ this additional hardware reduces the payload volume and substantially increases mission cost [NASA, 2008].

An alternative method of propellant gauging is to provide a known impulse to the spacecraft by a timed burn of thrusters. By measuring the craft's dynamic response to the impulse (acceleration 
and final velocity), an estimate of the spacecraft's total average mass during the burn can be calculated using Newton's Laws. By subtracting the fixed mass of the spacecraft, the mass of fuel left in the tanks can be estimated. This method has the distinct disadvantage of burning additional propellant to make the measurement.

Finally, burn-time integration is a book-keeping method in which careful records of all thruster firings are recorded and the total propellant usage is estimated via assumptions about the propellant use during each burn. This method requires no additional hardware but is subject to large uncertainties resulting from the guesswork involved in assumptions about operating performance in the thrusters.

Beginning in 2011, the Carthage Space Sciences group has worked on an alternative technique utilizing Experimental Modal Analysis (EMA) methods and real-time data reduction methods to gauge the volume of liquid in model spacecraft propellant tanks. Our previous work on parabolic flights has demonstrated viability of the technique and provided a rough estimate of the resolution in volume measurement possible with the technique [Mathe et al., 2012].

The short duration of microgravity available on parabolic flights (approximately 20 seconds per parabola) results in unsettled liquid sloshing continuously throughout the microgravity portions of the parabolic flight. In contrast, the propellant in a spacecraft such as a satellite in orbit around the earth, will generally be in a settled, non-sloshing state. To probe the resolution of the EMA method for settled fuel states, we proposed to the Wisconsin Space Grant Consortium to provide funding to implement the EMA gauging experiment on a sounding rocket where the smaller tank size and the $\approx 4$ minutes of microgravity result in a liquid in mechanical equilibrium with its containment vessel.

The RockSat-C program allows student-faculty teams to propose, design and build experiments of their own conception for flight on a Terrier-Orion sounding rocket at NASA's Wallops Flight Facility on Wallops Island, VA [Koehler, 2013]. Details of the Terrier-Orion flight profile are discussed in the following sections.

\section{Experimental Modal Analysis}

EMA involves the application of acoustic forces to test structures. Natural resonances of the test structure are excited by the applied force, and sensors affixed to the structure record the amplitude of the acoustic response across the range of resonating frequencies [Brandt, 2011].

The acoustic forces applied to the structure can be in the form of discrete impacts, continuous white-noise functions, or chirp functions. In the present experiment, we use broad-band white noise. A white noise spectrum consists of all frequencies within a specified range with each frequency component having the same spectral power. By introducing every possible driving frequency to the tank, we are guaranteed that the full set of structural resonant frequencies is present within the applied signal. Therefore, natural vibrational modes of the structure will be excited resulting in an increased amplitude in sensor response at the excited mode frequencies.

In order to record and isolate the resonant frequencies of the tank, two non-invasive sensors are 
attached to the tank. One sensor is located close to the actuator that provides the input spectrum of white noise frequencies. We refer to this sensor as the "monitor" as it generally reproduces the input spectrum with very little resonant mode amplification. A second sensor located far from the monitor produces an output that consists of the attenuated white noise spectrum with the resonant frequencies preferentially excited.

In the EMA method, Fast Fourier Transforms (FFT) of the monitor and sensor signals are computed and the ratio of sensor to monitor FFTs is derived to construct the Frequency Response Function (FRF). The FRF, in effect, normalizes the signal FFT by the monitor FFT and therefore isolates those frequency components that only appear in the signal sensor. In this way, the resonant modes of the tank are isolated and extracted from the applied white noise spectrum. Modal techniques can therefore be used as real-time diagnostics of structural properties. Fluid loading increases the effective mass of the loaded structure, resulting in a decrease in the structure's resonant frequencies. The essential experimental concept is represented in Fig. 1 .

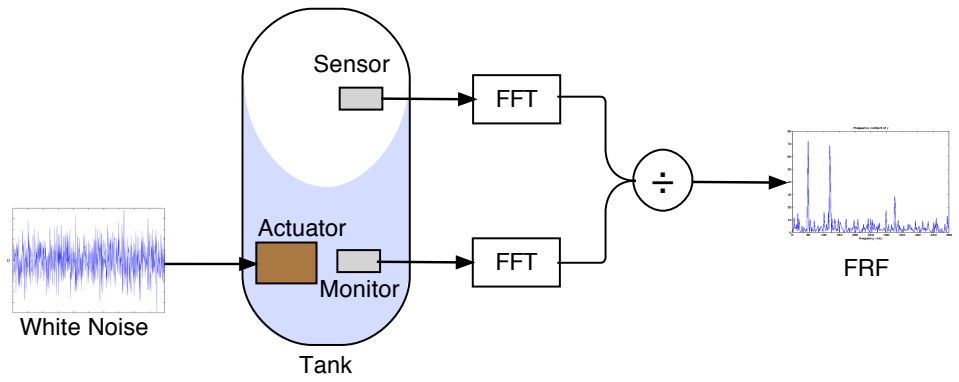

Figure 1: Experiment concept. A PZT patch actuator is adhered to a small experimental tank partially filled with water. Broadband white noise is introduced to the tank through the actuator. The Frequency Response Function is computed from the FFTs derived from the sensor and monitor signals.

\section{Experiment Objectives}

The central objective of the experiment is to continuously record signal and monitor response of a model propellant tank driven by a white noise spectrum throughout the duration of the TerrierOrion flight profile. Continuous recording of monitor and sensor signals satisfies the minimum success criteria for the project, but the larger analysis goal is to correlate shifts in the resonant mode frequencies with change in fill fraction. Because we have demonstrated fill-fraction resolution of better than 5\% with sloshing liquid in the parabolic flights, in the sounding rocket project in which we anticipated a settled fluid state, we hoped to push this resolution below $1 \%$ (to resolve fill fractions that differed by no more than $1 \%$ of the total tank volume).

\section{Sounding Rocket Mission Profile}

The Terrier Mk12-Improved Orion Sounding Rocket is configured with a first stage Terrier Mk12 motor providing $15037 \mathrm{lb}$ of thrust over a burn time of 5.2 seconds, a second stage Improved Orion motor with $8273 \mathrm{lb}$ of thrust over a burn time of 25.4 seconds, and two experiment bays with 9 payload canisters. The fifteen minute flight profile of the Terrier Orion rocket is illustrated 
in Fig. 2. The period of free-fall during which data acquisition occurs is between the end of the Orion stage burn at $T+40 \mathrm{sec}$. and $T+312 \mathrm{sec}$. Apogee for the flight was at 73 miles and time approximately $T+168 \mathrm{sec}$. The flight trajectory from launch Pad 1 at the Wallops Island Launch Facility is illustrated in Fig. 3 .

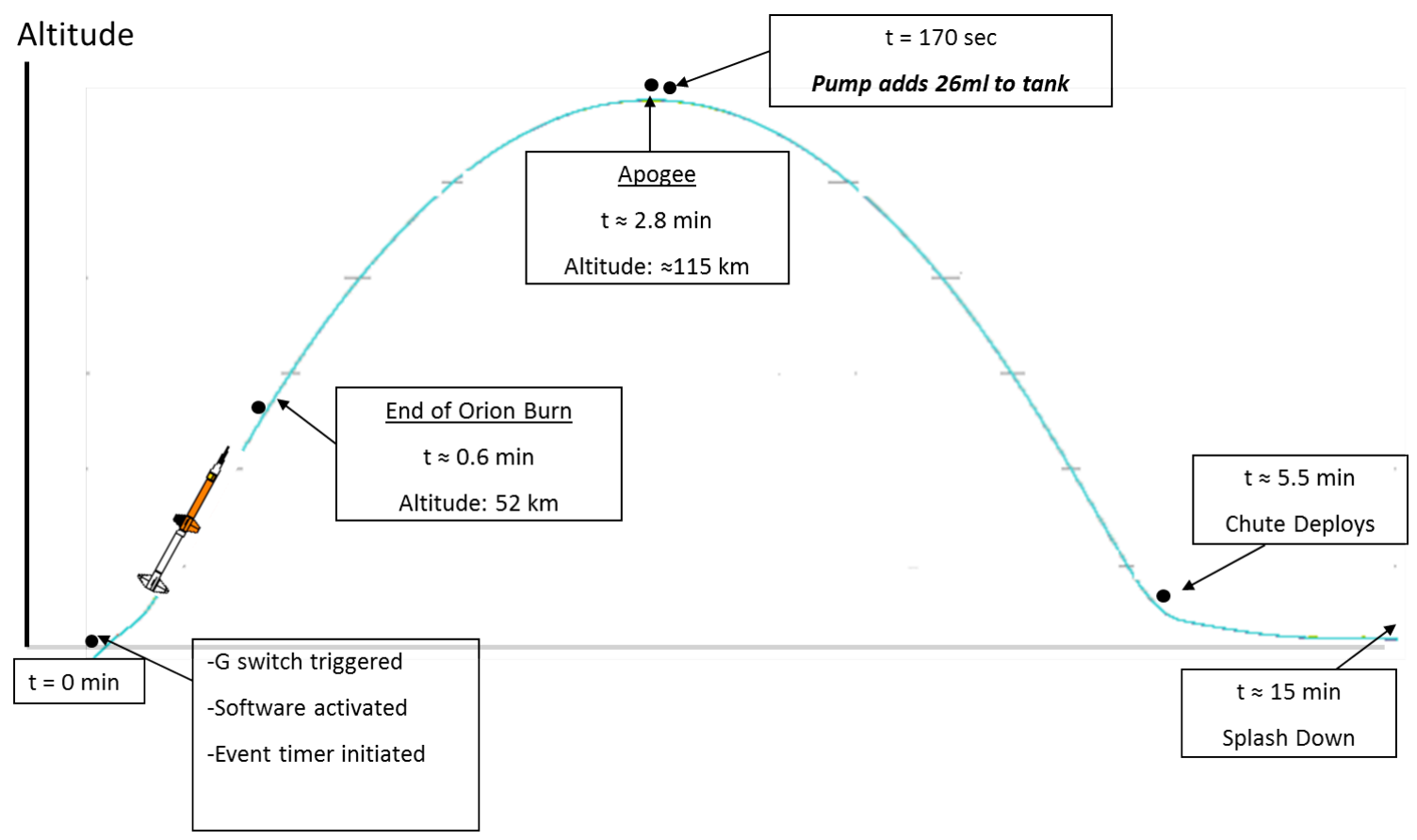

Figure 2: Terrier Orion Sounding Rocket flight profile

Launch accelerations exceed $25 \mathrm{~g}$ shortly after ignition of the first stage and the vehicle is moving at its maximum speed of $1300 \mathrm{~m} / \mathrm{s}$ at the end of second stage burnout. Payload separation from motors occurs at $T+125 \mathrm{sec}$. The vehicle launches from a launch rail set at $89^{\circ}$.

\section{Concept of Operations}

The concept of operation for the experiment is illustrated in Fig. 11. The experiment protocol has our $1419 \mathrm{ml}$ experimental tank initially filled with $160 \mathrm{ml}$ of water corresponding to a fill fraction of $11 \%$. Once in the zero-g portion of the flight trajectory, EMA data is continuously recorded. Approximately halfway through the flight trajectory, a solenoid valve opens and a micropump initiates the transfer of $26 \mathrm{ml}$ of water from a reservoir bladder into the experimental tank, providing a fill change of approximately $1.8 \%$ of the total tank volume. A flow sensor measures the total amount of liquid exchanged between fill bladder and tank.

\section{Experiment Design}

Our payload was divided into four subsystems: structural, electronics / software, hydraulic, and sensors. 


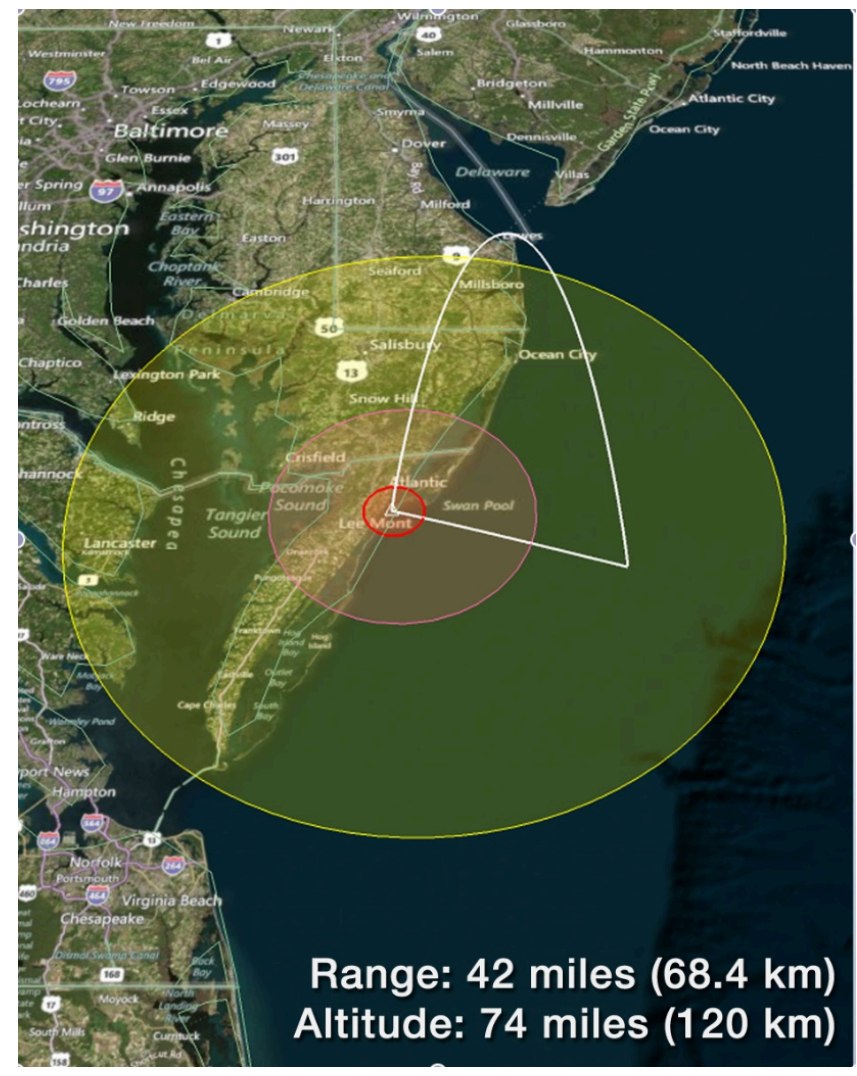

Figure 3: Sounding rocket flight trajectory from Wallops Island Flight Facility. 
The sensor subsystem consists of two identical PZT (lead zirconate titanate) transducers, one serving as the monitor and one as the signal sensor. An actuator driven by a piezo signal amplifier in turn driven by a white noise generator provides a broad band excitation signal to the experimental tank. A hydraulics subsystem consisting of solenoid valve, micro-pump and two fluid reservoirs exchanges liquid and air with the experimental tank. The electronics subsystem consists of two micro controllers, a white noise signal generator, a four channel analog-to-digital converter (ADC), a four channel digital-to-analog converter (DAC) for voltage and data conversions, and Compact Flash (CF) format data storage, as well as latch and activation circuits for experiment initiation. Each subsystem is detailed in brief below.

\section{Structural Subsystem}

The heart of the Structural Subsystem consists of a cylindrical tank of 5.5 inch diameter and of 4 inch height. This experimental tank holds the water (our propellant simulant) and has the PZT transducers affixed to the outside surface. Two 1/4-inch NPT ports are located on top of the tank for filling and draining. Above the tank rests a deck, which we call the hanger, for holding hydraulic components including the pump, solenoid valves and water bladders. The hanger is supported by pillars, which we call secondary standoffs, in order to mechanically isolate the tank from the rest of the structure. A secondary containment vessel envelops the tank and hanger. Mounted above the secondary containment vessel is a polycarbonate plate to hold the electronic components. Finally, the RockSat payload canister which serves as the external sheath to the payload is bolted to the electronics plate by five standoffs. The canister is also bolted to the bottom of the secondary containment baseplate.

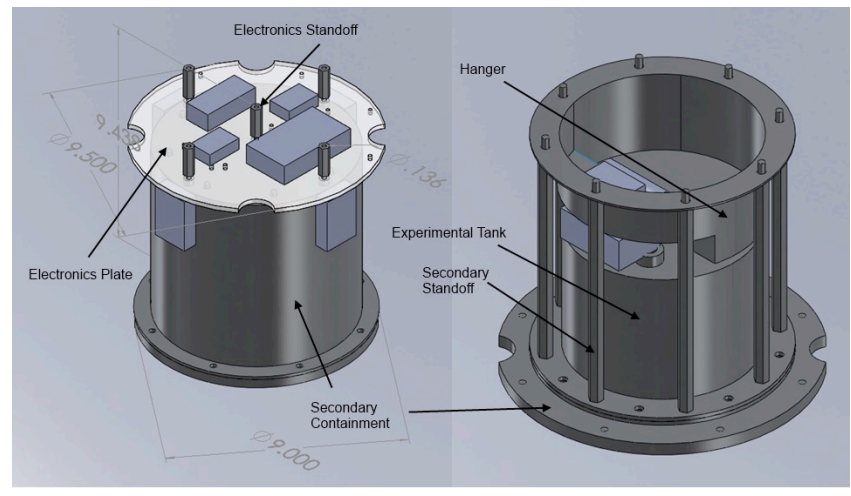

Figure 4: Structural subsystem consisting of experimental tank, electronics deck, support structure and secondary containment vessel.

\section{Sensor Subsystem}

The sensors and the actuator (also a PZT transducer) are affixed to the experimental tank by means of a thin double-sided adhesive. The sensors convert mechanical vibrations of the tank to proportional voltage signals at the level of $100 \mathrm{~s}$ of $\mathrm{mV}$. 
The actuator is driven by a white noise signal amplified by a compact piezo driver that steps up the TTL signal from the white noise generator to a proportional signal with an RMS voltage of \pm 200V.

\section{Electronics Subsystem}

The main functions of the Electronics Subsystem are to send a pre-recorded white noise signal to the actuator, to store signals from the sensor and monitor to a flash disk, and to control the event timer for the pump and solenoid valve. The electronics are powered by four $6 \mathrm{~V}$ nickel metal hydride (NiMH) rechargeable battery packs wired in a series of two parallel circuits to produce an output voltage of $12 \mathrm{VDC}$.

The latch circuit board regulates the power activation. In accordance with the Wallops Flight Facility Sounding Rocket Program Guidelines, activation occurs when both Wallops Flight Facility shorts the remove-before-flight (RBF) connection and when the g-switch is activated by launch acceleration. Power is then distributed to the relay circuits, white noise amplifier, and the 12 to $5 \mathrm{~V}$ and 12 to $3 \mathrm{~V}$ power regulators.

Upon activation of the g-switch by launch accelerations, the TIP triggers the mini SD card player. A prerecorded .ad4 file containing reference tones and the white noise signal is sent from the SD card player to the Piezo Amplifier for distribution to the actuator PZT. The signals from the sensor and monitor are processed by a separate micro controller (a Tern B-Engine) that handles on-board analog-to-digital conversion and data storage via a Compact Flash (CF) card. The B-Engine has an onboard ADC that samples at a rate of 32,000 samples per second. The TIP also regulates the timing of fill changes and handles event timing for the duration of the flight. Software development for both TIP and B-Engine was in C.

At sample rates of $32 \mathrm{kHz}$, The Nyquist frequency for the experiment is $16 \mathrm{kHz}$, well above the highest frequencies $(5 \mathrm{kHz})$ reliably detected in the resonance spectrum of the experimental tank.

\section{Hydraulics Subsystem}

The key elements of the hydraulics chain are illustrated in Fig. 5. A flexible bladder containing $50 \mathrm{ml}$ of water is connected by tubing to a flow totalizer, solenoid valve, flow pump and to the experimental tank. An identical flexible bladder is used to equilibrate pressure in the tank during fill-changes.

The tank itself has two 1/4-inch NPT ports for connection to bladder and reservoir. A $24 \mathrm{~V}$ micropump transfers water at a rate of around 1.0 liter/min. between bladder and tank. The pump is activated simultaneously with the opening of a solenoid valve. Both the valve and the pump remain powered for $\approx 2$ seconds to transfer the $26 \mathrm{ml}$ of water from bladder to tank.

The integrated payload experiment is shown in Fig. 6. Fully assembled and loaded with water, the payload weighs $20.0 \mathrm{lb}$ and fully occupies one payload canister. 


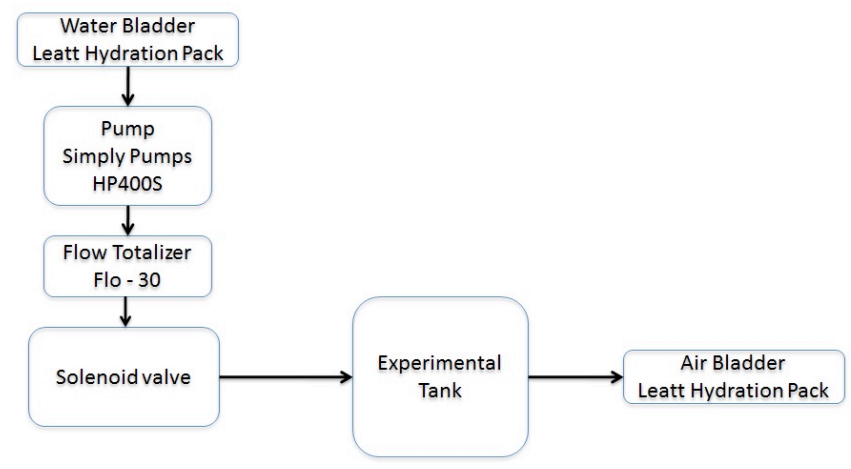

Figure 5: Hydraulic flow loop.

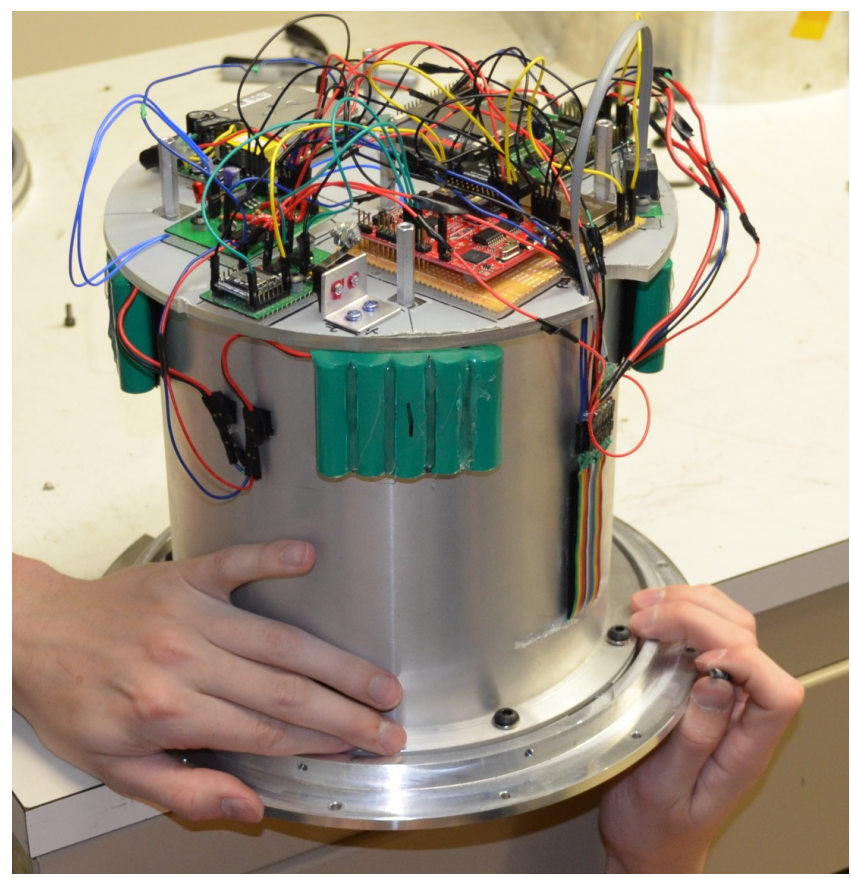

Figure 6: Fully assembled payload canister before wire assemblies are staked down for launch and RockSat canister is attached. 


\section{Flight and Ground Data}

Prior to developing the sounding rocket payload experiment, the authors flew EMA fuel gauging experiments on parabolic aircraft using a larger cylindrical tank of volume 7.5 liters. The resonant mode peaks in the larger tank are distinguishable with a frequency resolution of around 1-2 $\mathrm{Hz}$ - at the limit of our sampling resolution. In contrast, the mode structure for the sounding rocket payload experiment has a lower frequency resolution of around $20 \mathrm{~Hz}$. Fig. 7 shows a typical FRF obtained from the sounding rocket payload from which we can identify three primary modes which we label from lowest frequency to highest P1, P2, P3.

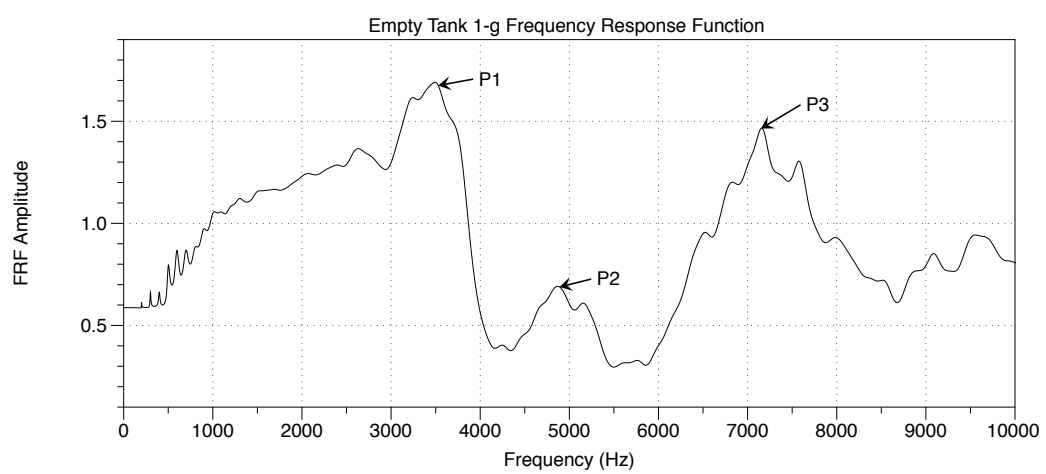

Figure 7: Sample Frequency Response Function for the empty tank. The frequencies P1, P2 and P3 were tracked throughout ground testing. Peak P2 was tracked through across two fill levels during flight.

The dependence of resonant mode P2 on fill level is shown in Fig. 8 for both ground (1-g) testing and for the two fill fractions used in the sounding rocket flight. Error bars for the ground data are standard errors obtained over a dozen sampling windows at each fill fraction. Standard errors for the flight data are not defined because of the small number of samples taken in flight.

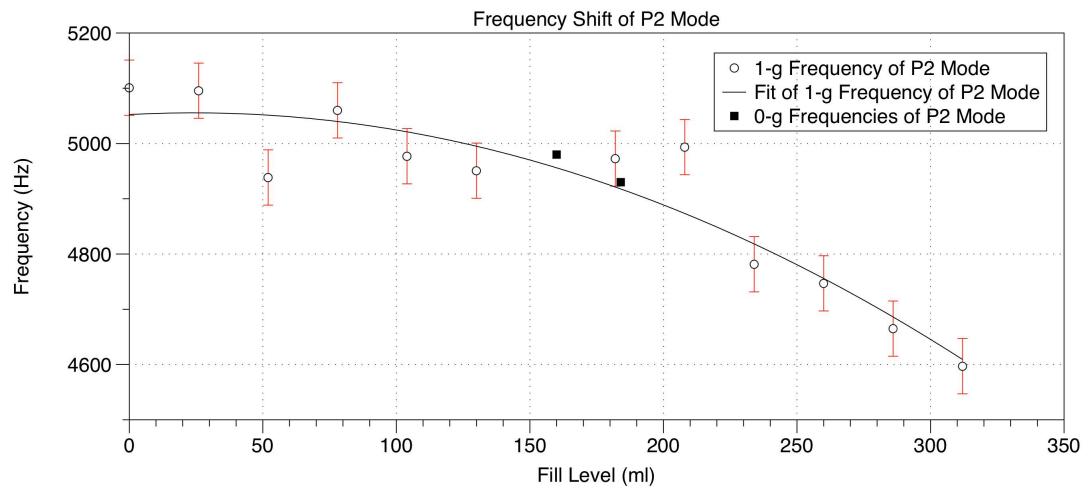

Figure 8: Cumulative data for peak frequencies as a function of fill level for both ground testing and for the two fill levels obtained during sounding rocket flight. Error bars are obtained from standard errors in ground testing data.

The relatively brief flight of the sounding rocket does not afford the opportunity to explore a large number of fill changes, but the flight data are suggestive, nonetheless. Mode shifts at the two fill levels obtained in zero-g do follow the anticipated trend. Mass-loading of the tank structure as a 
result of added liquid should indeed result in decreasing resonant mode frequency as fill level is increased and this is what is observed in both ground and flight data.

\section{Summary and Conclusions}

EMA is an effective technique in real-time tracking of resonant mode shifts as a result of filllevel changes in a tank under arbitrary gravity states. Previous work has established that the EMA method can be used to infer fill-level changes of less than a few percent of tank volume under sloshing, zero-g conditions. The present study demonstrates that fill fraction changes of around $5 \%$ are potentially resolvable in a small cylindrical tank under zero-g conditions. The sounding rocket payload is also subject to spin about its vertical (symmetry) axis at a rate of about $5 \mathrm{~Hz}$, and no confounding effects of this spin state were observed in the flight data.

EMA is a robust and viable method of inferring liquid volume in a spacecraft propellant tank in zero-g and has several advantages over existing methods of propellant gauging: EMA-based gauging is non-invasive, requires no additional hydraulics and can be performed in real-time with minimal computational overhead. Propellant tanks with integrated PZT sensors and minimal signal processing systems may one day replace the bulky pressurized systems currently in use in most gauging-critical spacecraft systems.

\section{Acknowledgments}

The authors gratefully acknowledge the Wisconsin Space Grant Consortium for financial support. The authors also acknowledge the useful contributions made by Prof. Brant Carlson.

\section{References}

[NASA, 2005] NASA Exploration Systems Architecture Study Final Report, available at http: //www.nasa.gov/exploration/news/ESAS_report.html.

[NASA, 2008] Advanced Space Transportation Program: Paving the Highway to Space, available at http://www.nasa.gov/centers/marshall/news/background/facts/astp.html.

[Mathe et al., 2012] Mathe, S., Anderson, K., Bakkum, K., Lubick, L., Robinson, J., Weiland, D., Werlink, R., Crosby, K.M. (2012). Modal Evaluation of Fluid Volume in Spacecraft Propellant Tanks. Proceedings of the 22nd Annual Wisconsin Space Conference.

[Koehler, 2013] RockSat Student Payload Experiment Program (2013). Information available at http://spacegrant. colorado.edu/national-programs/rs-c-2014-home.

[Brandt, 2011] Brandt, A. (2011). Noise and vibration analysis: Signal analysis and experimental procedures. Hoboken NJ: Wiley. 